\title{
Comparison of Growth Performance of Goat Kids Under Supplementation with Different Probiotics
}

\author{
K. Smita Sivadasan* and Shinoj Subramannian \\ ICAR-Krishi Vigyan Kendra of Central Marine Fisheries Research Institute, Ernakulam, Kerala, INDIA \\ *Corresponding author: KS Sivadasan; E-mail: drsmitavet@gmail.com
}

Received: 30 July, 2020

Revised: 23 Sept., 2020

Accepted: 28 Nov., 2020

\begin{abstract}
This study was conducted to assess the comparative growth performance of goat kids supplemented with yeast as individual probiotic and yeast based combined probiotic preparation. Fifteen male crossbred Malabari goat kids of three months age and having body weights in the range of $8.5 \mathrm{~kg}$ to $10.5 \mathrm{~kg}$ were selected, dewormed and divided randomly into three treatment groups each having 5 animals (Average body weight of each group being 9.5-9.6 kg). The first group of goats (T1) was kept as control and was not supplemented with any probiotics. The second group of goats (T2) was supplemented with yeast, Saccharomyces cerevisiae while the third group (T3) was supplemented with a Saccharomyces cerevisiae based combined probiotic containing Saccharomyces cerevisiae, Saccharomyces boulardii, Lactobacillus acidophilus and Propionibacterium freudenreichii. The final body weights of both the probiotic supplemented kids (T2 and T3) were significantly $(\mathrm{P}<0.05)$ higher than unsupplemented kids in control (T1) group. The body weight among both the yeast (T2) and Saccharomyces cerevisiae based combined probiotic supplemented groups also differed significantly $(\mathrm{P}<0.05)$. Similarly, goat kids fed Saccharomyces cerevisiae based combined probiotic preparation had higher average daily weight gain over individual yeast fed and control goats.
\end{abstract}

\section{HIGHLIGHTS}

(0 Growth performance of goat kids supplemented with different yeast based probiotics.

( Body weights of goat kids from both probiotic preparation supplemented groups found to be significantly higher than control group.

Keywords: Body weight, Probiotics, Yeast, Goat kids

There is a general concern on the widespread use of antibiotics as growth promoters in animal and poultry feed. For several decades, antibiotics and chemotherapeutic agents in prophylactic dosages have been used in animal feed to improve animal welfare and to obtain economic benefits in terms of improved animal performance and reduced medication costs. However, there are increasing concerns about the risk of developing cross-resistance and multiple antibiotic resistances in pathogenic bacteria in both human and livestock linked to the therapeutic and sub therapeutic use of antibiotics in livestock (Vandana et al., 2013). Probiotics are known as safe alternatives to antibiotics. They are defined as "live microorganisms that may beneficially affect the host upon ingestion by improving the balance of the intestinal microflora" (Fuller,
1989). Intensive rearing conditions may induce stress and can cause changes in the composition of the intestinal microflora that reduce the animals' resistance to infection. The benefits of probiotics in farm animals are improved resistance to disease, improved feed intake, enhanced feed conversation rate, improved daily weight gain and total body weight gain in chicken, pig, sheep, goat and cattle (Musa et al., 2009). The principal microorganisms used as probiotics for ruminants are bacteria and yeasts. Most commercially available probiotics are certain strains of

How to cite this article: Sivadasan, K.S. and Subramannian, S. (2020) Comparison of growth performance of goat kids under supplementation with different probiotics. J. Anim. Res., 10(6): 1063-1065.

Source of Support: None; Conflict of Interest: None 
Lactobacillus spp, Bifidobacterium spp, Enterococcus spp and yeasts as individual or combined preparations. The present study was undertaken with the objective of comparing the effect of individual probiotic containing Saccharomyces cerevisiae and Saccharomyces cerevisiae based combined probiotic supplementation for the body weight gain in goat kids.

\section{MATERIALS AND METHODS}

The trial was conducted in farmers fields located in Nayarambalam and Cherai village of Ernakulam district, Kerala, India for a period of eight fortnights. Fifteen male crossbred Malabari goat kids of three months age and having body weights in the range of $8.5 \mathrm{~kg}$ to $10.5 \mathrm{~kg}$ were selected, dewormed and divided randomly into three treatment groups each having 5 animals (Average body weight of each group being 9.5-9.6 kg). The first group of goats (T1) was kept as control and was not supplemented with any probiotics. The second group of goats (T2) was supplemented with yeast, Saccharomyces cerevisiae while the third group (T3) was supplemented with a Saccharomyces cerevisiae based combined probiotic containing Saccharomyces cerevisiae, Saccharomyces boulardii, Lactobacillus acidophilus and Propionibacterium freudenreichii. Yeast and Saccharomyces cerevisiae based combined probiotic preparation was fed to the animals through concentrate feeds at the rate of $1 \mathrm{gm}$ per $\mathrm{kg}$ of concentrate feed. While introducing them, initially a pinch of yeast or probiotic preparation was added to the feed to accustom them to the change. All the animals were fed equal amount of concentrate feed having $14 \%$ DCP and 70\% TDN along with coconut meal cake-80-100 g/animal and adlibitum local green grasses and jackfruit tree leaves. Gradually, the amount was increased to reach the required levels of feeding. Body weight was measured at fortnightly intervals and average for each group was calculated at the end. The data obtained in the trial were analyzed statistically using One way ANOVA and Tukey's Honest significant difference (HSD) test (Snedecor and Cochran, 1994).

\section{RESULTS AND DISCUSSION}

The final body weights obtained after the 8 fortnights trial was $13.32 \pm 0.29 \mathrm{~kg}$ in unsupplemented control (T1), 14.88 $\pm 0.29 \mathrm{~kg}$ in Saccharomyces cerevisiae supplemented group (T2) and $16.20 \pm 0.13 \mathrm{~kg}$ in Saccharomyces cerevisiae based combined probiotic supplemented (T3) group. The statistical analysis indicated that the final body weights of both the probiotic supplemented kids (T2 and T3) were significantly $(\mathrm{P}<0.05)$ higher than unsupplemented kids in control (T1) group. The body weight among both the yeast (T2) and Saccharomyces cerevisiae based combined probiotic supplemented groups also differed significantly $(\mathrm{P}<0.05)$. This is in agreement with the findings of many workers (Jinturkar et al., 2009) who observed that supplementation of Lactobacillus acidophillus and Saccharomyces cerevisiac individually and in combination produced higher final body weights in Osmanabadi goats as compared to the unsupplemented control group and also the body weight of group fed combined probiotics had higher body weight than individually fed animal group or control group. Reports (Kochewad et al., 2009) also showed that Osmanabadi kids which received combined probiotics supplementation were found to have increased body weight and growth parameters as compared to the non supplemented kids. Researchers (Jayabal et al.,

Table 1: Body weight in $\mathrm{kg}($ Mean \pm S.E. $)$ measured at fortnightly intervals

\begin{tabular}{llll}
\hline Fortnightly intervals of measurement & T1 $^{*}$ & T2 $^{\#}$ & T3 $^{\# \#}$ \\
\hline At the start of trial-0 weeks & $09.60 \pm 0.45$ & $9.56 \pm 0.35$ & $9.66 \pm 0.40$ \\
2 weeks & $10.14 \pm 0.39$ & $10.46 \pm 0.30$ & $10.84 \pm 0.29$ \\
4 weeks & $10.94 \pm 0.41$ & $11.86 \pm 0.43$ & $12.40 \pm 0.44$ \\
6 weeks & $11.64 \pm 0.43$ & $12.50 \pm 0.48$ & $13.52 \pm 0.29$ \\
8 weeks & $12.18 \pm 0.30$ & $13.58 \pm 0.38$ & $14.76 \pm 0.24$ \\
10 weeks & $12.78 \pm 0.30$ & $14.18 \pm 0.31$ & $15.36 \pm 0.20$ \\
12 weeks & $13.32 \pm 0.29$ & $14.88 \pm 0.29$ & $16.20+0.13$ \\
\hline
\end{tabular}

*T1 - Control; \#T2 - Saccharomyces cerevisiae supplemented group; \#\# T3 - Saccharomyces cerevisiae based combined probiotics supplemented group. 
2008) who assessed the growth performance of stall fed non-descript goats on probiotics supplementation found that the body weight gain of probiotic supplemented kids was significantly $(\mathrm{P}<0.05)$ higher than the control kids but the difference among probiotic supplemented groups were non-significant which is partially different from the present study.

Further, the average daily body weight gain (ADG) in unsupplemented control (T1) was $0.044 \mathrm{~g}$ /day whereas both probiotic supplemented groups had higher average daily gains. The Saccharomyces cerevisiae supplemented group (T2) had ADG of $0.063 \mathrm{~g} /$ day while the Saccharomyces cerevisiae based combined probiotic supplemented (T3) group had $0.078 \mathrm{~g} /$ day gain. This is in agreement with article (Jinturkar et al., 2009) which reported that the Osmanabadi goat kids which were fed combined probiotic preparation had higher average daily weight gain over individual probiotic fed and control goats. Researchers (Singh et al., 1998) also found that the crossbred calves supplemented with Saccharomyces cerevisiae had higher average daily weight gain than control calves.

\section{CONCLUSION}

Though body weight gain was slightly higher in Saccharomyces cerevisiae based combined probiotic supplemented kids, on account of its easy availability, both yeast alone or in combination can be effectively used as a probiotic supplement. Probiotic supplementation in goat kids significantly increases their body weight.

\section{REFERENCES}

Fuller, R. 1989. Probiotics in man and animals. J. Appl. Bacteriol., 66: 365-378.

Jayabal, T., Murallidharan, R., Gnanaraj, P.T. and Murugan, M. 2008. Growth performance of stall-fed goats under probiotic supplementation. Indian J. Vet. Anim. Sci., 4(5): 179-184.

Jinturkar, A.S., Gujar, B.V., Chauhan, D.S. and Patil, R.A. 2009. Effect of feeding probiotics on the growth performance and feed conversion efficiency in goat. Indian J. Anim. Res., 43(1): 49-52.

Kochewad, S.A., Chahande, J.M., Kanduri, A.B., Deshmukh, D.S., Ali, S.A. and Patil, V.M. 2009. Effect of probiotic supplementation on growth parameters of Osmanabadi kids. Vet. World, 2(1): 29-30.

Musa, H.H., Wu, S.L., Zhu, C.H., Seri, H.I. and Zhu, G. Q . 2009. The potential benefits of probiotics in animal production and health. J. Anim. Vet. Adv., 8(2): 313-321.

Rai, V., Yadav, B., Lakhani, G.P. 2013. Application of probiotic and prebiotic in animals production: A review. Environ. Ecology, 31(2B): 873-876.

Snedecor, G.W. and Cochran, W.G. 1994. Statistical methods, $8^{\text {th }}$ Edn., Oxford and IBH Publications, New Delhi.

Singh, R., Chaudhary, L.C., Kamra, D.N. and Pathak, N.N. 1998. Effect of dietary supplementation with yeast cell suspension (Saccharomyces cerevisiae) on nutrient utilization and growth response in crossbred calves. AJAS, 11(3): 268-271. 
